\title{
Adjuvant chemotherapy provided survival benefit for stage T2N0 gastric cancer with high-risk factors
}

\author{
Z. WANG ${ }^{1}$, J. YAN ${ }^{1}$, W. HU ${ }^{1, *}$, J. ZHANG ${ }^{2}$, B. HUO ${ }^{3}$ \\ ${ }^{1}$ Department of Chemoradiotherapy, Tangshan People's Hospital, Tangshan, PR China; ${ }^{2}$ Department of Radiation Oncology, Shandong Cancer \\ Hospital, Shandong Academy of Medical Sciences, Jinan, PR China; ${ }^{3}$ Department of Traditional Chinese Medicine, The Fourth Hospital of Hebei \\ Medical University, Shijiazhuang, PR China
}

${ }^{*}$ Correspondence: tssrmyy_hu@163.com

Received June 20, 2017 / Accepted October 4, 2017

\begin{abstract}
The value of adjuvant chemotherapy in T2N0 gastric cancer (GC) remains controversial. The aim of this retrospective study is to define a high-risk subgroup of pathological T2N0 GC patients and examine the impact of adjuvant chemotherapy on overall survival (OS). A total of 225 patients underwent R0 resection for T2N0 gastric adenocarcinoma between 2002 and 2012 and 51/225 (22.7\%) of these received adjuvant chemotherapy. Multivariate Cox regression identified tumor location in the Upper $1 / 3$ of the stomach $(\mathrm{p}<0.001)$, larger tumor diameter $(\mathrm{p}=0.013)$, lymphatic and/or blood vessel invasion $(\mathrm{p}=0.001)$, and perineural invasion $(\mathrm{p}<0.001)$ as independent risk factors associated with significantly decreased OS. There were 141 patients with at least one risk factor who were defined in the high-risk subgroup. After propensity score matching, there was a significant trend toward improved OS ( $\mathrm{p}=0.042)$ with adjuvant chemotherapy in the high-risk subgroup. In conclusion, we identified a high-risk subgroup of T2N0 GC with at least one of the independent risk factor listed above, and we found that adjuvant chemotherapy significantly improved OS for this subgroup.
\end{abstract}

Key words: gastric cancer, adjuvant chemotherapy, prognosis, propensity score matching

Gastric cancer (GC) is an aggressive malignancy with poor prognosis. It is the fourth common malignant tumor and third most common cause of cancer deaths worldwide [1]. The primary treatment for curing gastric cancer without distant metastases is radical gastrectomy and lymphadenectomy. In several randomized trials, fluoropyrimidine-based adjuvant chemotherapy was shown to improve survival in patients with T3, T4, and/or node-positive GC [2-4]. In the JCOG 8801 study, curative surgery alone yielded a very good survival rate in patients with T1 cancer [5]. However, several retrospective population-based studies found no overall benefit for adjuvant therapy in stage I gastric cancer [6-8] and adjuvant treatment was not recommended for T1N0 GC.

The value of adjuvant chemotherapy also remains controversial in T2N0 GC due to lack of evidence from randomized clinical trials. Although the overall prognosis of T2N0 GC is favorable, several retrospective studies have identified some poor-prognostic subgroups who also carried high-risk factors. These included high grade and large tumors, older age and lymphatic vessel and nerve invasion $[9,10]$. These high-risk factors were not only demonstrated to be indepen- dent poor prognostic markers for survival, but also markers for adjuvant chemotherapy in various kinds of solid tumor, such as non-small cell lung cancer [11], colorectal cancer [12] and breast cancer [13]. It is believed that the high-risk groups from T2N0 GC potentially benefit from adjuvant chemotherapy. The aim of this retrospective study is to identify stage T2N0 GC patients at high risk of recurrence or death, and analyze if these high-risk patients benefit from adjuvant chemotherapy.

\section{Patients and methods}

Patient selection. Inclusion criteria were as follows: (1) all patients received radical gastrectomy (R0 resection) with lymphadenectomy; (2) all had pathologically confirmed T2N0 gastric adenocarcinoma, with no clinical evidence of distant metastasis (M0); (3) all had adequate major organ function, including cardiac, hepatic, and renal function and (4) all had follow-up information at least once after resection.

The exclusion criteria were: (1) patients who received any neo-adjuvant therapy before resection and (2) those with 
an additional synchronous or metachronous carcinoma. All tumor pathologic staging was based on the seventh edition of the American Joint Committee on Cancer (AJCC) tumornode-metastasis (TNM) classification.

Surgical procedure. The recommended surgical procedure is complete excision of the tumor and extended lympnectomy (D2). This includes resection of perigastric lymph nodes (D1) and also lymph nodes along the left gastric, common hepatic, splenic and coeliac arteries. Lymphadenectomy greater than D1 but less than D2 is named D1 plus.

Selection of patient characteristics. Clinical-pathologic characteristics were grouped in categorical variables for analysis. Those covariates included age $(<50$ and $\geq 50)$, gender (male and female), tumor diameter $(\leq 3 \mathrm{~cm}$ and $>3 \mathrm{~cm})$, histological type (differentiated and undifferentiated), lymphatic and/or blood vessel invasion (LBVI negative and positive), perineural invasion (PNI negative and positive), resection type (subtotal and total), lymph node dissection (D2 and D1 plus) and lymph nodes examination $(\geq 15$ and $<15)$.

Statistical analysis. The primary endpoint of this study was overall survival (OS); defined as the time from the date of surgery to the date of death. The differences in clinicalpathologic characteristics between groups were assessed by $\chi^{2}$ test or Fisher's exact test. The Kaplan-Meier method compared OS between groups and log-rank tests were used as significance tests. Cox regression identified the independent outcome predictors and further analysis was performed in patients with at least one high risk factor to determine if adjuvant chemotherapy provided survival benefit. To further adjust for confounding variables, propensity score-matched analyses were performed to compare the survival outcomes between the treatment groups (Group S and Group SC). Before matching, all patient and tumor clinical variables were compared by the $\chi^{2}$ test and the imbalanced variables were included in multivariate logistic regression for propensity score. Two-to-one matching without replacement was completed using the nearest-neighbor match on the logit of the propensity score for treatment approach. This is derived from age, sex, tumor diameter, histological type, LBVI, PNI, resection type and lymph node dissection. The caliper width was set at 0.2 times the standard deviation of the propensity score.

The Kaplan-Meier method then estimated survival as a function of time, and the survival differences were analyzed by log-rank test. Multivariate survival analysis was not performed because of the limited high risk group sample size. All tests were two-sided, and $\mathrm{p}<0.05$ was considered statistically significant. Analysis was performed with the SPSS statistical software package (SPSS, Inc., Chicago, IL, USA), version 19.0.

\section{Results}

Patient characteristics. In this retrospective study, 3,752 patients with GC who consecutively underwent gastrectomy at our hospitals between January 2002 and December 2012 were reviewed, and after a thorough search, 225 patients with T2N0M0 gastric cancer were included in the final analysis (Table 1). Of these patients, $77.3 \%(n=174)$ were treated with surgery alone (Group S), and 22.7\% ( $\mathrm{n}=51)$ underwent adjuvant chemotherapy after surgery (Group SC). Compared Patients in the S Group had significantly larger and more poorly differentiated tumors than those in the Sc group. They were greater than $3 \mathrm{~cm}$ in size and always had more LBVI and PNI (Table 1).

Analysis of independent risk factors. The median followup interval was 78 months and the overall 5-year survival rate was $75.7 \%$. Univariate analysis identified the following factors with prognostic value: tumor location and diameter, LBVI and PNI. Multivariate Cox regression then demonstrated that the risk factors in univariate analysis were also independent risk factors. Patients with tumors in the upper

Table 1. Patient clinical characteristics.

\begin{tabular}{|c|c|c|c|c|}
\hline Variable & All Cohort & No aCHT & aCHT & p-value \\
\hline No. of patients & 225 & 174 & 51 & \\
\hline Age (years) & & & & 0.416 \\
\hline$<50$ & $44(19.6)$ & $32(18.4)$ & $12(23.5)$ & \\
\hline$\geq 50$ & $181(80.4)$ & $142(81.6)$ & $39(76.5)$ & \\
\hline Gender & & & & 0.419 \\
\hline Male & $162(72.0)$ & $123(70.7)$ & $39(76.5)$ & \\
\hline Female & $63(28.0)$ & $51(29.3)$ & $12(23.5)$ & \\
\hline Tumor location & & & & 0.456 \\
\hline Upper1/3 & $42(18.7)$ & $34(19.5)$ & $8(15.7)$ & \\
\hline Middle1/3 & $56(24.9)$ & $40(23.0)$ & $16(31.4)$ & \\
\hline Lower $1 / 3$ & $127(56.4)$ & $100(57.5)$ & $27(52.9)$ & \\
\hline Tumor diameter & & & & 0.001 \\
\hline$\leq 3 \mathrm{~cm}$ & $120(53.3)$ & $103(59.2)$ & $17(33.3)$ & \\
\hline$>3 \mathrm{~cm}$ & $105(46.7)$ & $71(40.8)$ & $34(66.7)$ & \\
\hline Histological type & & & & 0.038 \\
\hline Differentiated & $117(52.0)$ & 97(55.7) & $20(39.2)$ & \\
\hline Undifferentiated & $108(48.0)$ & $77(44.3)$ & $31(60.8)$ & \\
\hline LBVI & & & & 0.004 \\
\hline Negative & 201(89.3) & $161(92.5)$ & $40(78.4)$ & \\
\hline Positive & $24(10.7)$ & $13(7.5)$ & $11(21.6)$ & \\
\hline PNI & & & & 0.004 \\
\hline Negative & $207(92.0)$ & $163(93.7)$ & $44(86.3)$ & \\
\hline Positive & $18(18.0)$ & $11(6.3)$ & $7(13.7)$ & \\
\hline Resection type & & & & 0.156 \\
\hline Subtotal & 205(91.1) & $156(89.7)$ & $49(96.1)$ & \\
\hline Total & $20(8.9)$ & $18(10.3)$ & $2(3.9)$ & \\
\hline Lymph node dissection & & & & 0.517 \\
\hline D2 & $128(56.9)$ & $101(58.0)$ & $27(52.9)$ & \\
\hline D1 plus & $97(43.1)$ & $73(42.0)$ & $24(47.1)$ & \\
\hline $\begin{array}{l}\text { Total number of lymph } \\
\text { nodes examined }\end{array}$ & & & & 0.185 \\
\hline$\geq 15$ & $111(49.3)$ & $90(51.7)$ & $21(41.2)$ & \\
\hline$<15$ & $114(50.7)$ & $84(48.3)$ & $30(58.8)$ & \\
\hline
\end{tabular}

Abbreviations: LBVI, lymphatic and/or blood vessel invasion; PNI, perineural invasion 
third of the stomach had greater risk of death compared to other sites, (HR 3.97, 95\% CI 2.09-7.52, p<0.001). Mortality hazard also increased for patients with larger tumor (HR, 1.99 95\%CI, 1.16-3.43; $\mathrm{p}=0.013$ ), LBVI (HR, 3.85 95\%CI, 1.75-8.48; $\mathrm{p}=0.001$ ), and PNI (HR, 4.03 95\%CI 1.84-8.83; $\mathrm{p}<0.001)$. These results are listed in Table 2.

Analysis of patients undergoing different chemotherapy. While 6 SC patients (11.8\%) received fluorouracil monotherapy, the 45 (88.2\%) who had combined chemotherapy regimen obtained greater OS benefit $(\mathrm{HR}=0.211$; 95\%CI: $0.063-0.710 ; \mathrm{p}=0.012)$; and thirty-eight $(84.4 \%)$ of these were treated with fluorouracil combined with platinum and $7(15.6 \%)$ had taxane combined with platinum. No differences in OS were observed between the two subgroups $(\mathrm{HR}=1.142$; 95\%CI: $0.250-5.213 ; \mathrm{p}=0.864)$. Treatment was continued for at least 3 cycles in 46 patients $(90.2 \%)$; and patients who withdrew from treatment did so because of adverse events, detection of metastasis or relapse. In addition, patients who received 3 or more chemotherapy cycles had better OS than those who had less. (HR $=0.265$; 95\%CI: 0.071 0.985; $\mathrm{p}=0.048$ ); and survival analysis is shown in Table 3 .

Analysis of risk factor groups. Four independent OS risk factors were identified in this study cohort. Patients with at least one risk factor were defined as high-risk group, and those without risk factor were low-risk group. There were 141 patients in the high-risk group and these had significantly lower OS (HR 2.86, 95\% CI 1.58-5.18, p <0.001; Figure 1).

Significant histological-type differences were also found in the $S$ and SC high risk Groups $(\mathrm{p}=0.019)$ and LBVI $(\mathrm{p}<0.017)$, and propensity score-matched analyses with $2: 1$

Table 2. Univariate and multivariate analyses of OS prognostic factors.

\begin{tabular}{|c|c|c|c|c|c|c|}
\hline \multirow{2}{*}{ Variable } & \multicolumn{3}{|c|}{ Univariable Analysis } & \multicolumn{3}{|c|}{ Multivariable Analysis } \\
\hline & HR & $95 \% \mathrm{CI}$ & p-value & HR & $95 \% \mathrm{CI}$ & p-value \\
\hline \multicolumn{7}{|l|}{ Age (years) } \\
\hline$<50$ & 1.00 (ref.) & & & 1.00 (ref.) & & \\
\hline$\geq 50$ & 1.69 & $0.84-3.41$ & 0.144 & 1.60 & $0.78-3.29$ & 0.203 \\
\hline \multicolumn{7}{|l|}{ Gender } \\
\hline Male & 1.00 (ref.) & & & 1.00 (ref.) & & \\
\hline Female & 1.10 & $0.64-1.88$ & 0.731 & 1.54 & $0.85-2.80$ & 0.157 \\
\hline \multicolumn{7}{|l|}{ Tumor location } \\
\hline Lower $1 / 3$ & 1.00 (ref.) & & & 1.00 (ref.) & & \\
\hline Middle1/3 & 1.40 & $0.78-2.52$ & 0.267 & 1.15 & $0.60-2.22$ & 0.672 \\
\hline Upper $1 / 3$ & 2.31 & $1.29-4.12$ & 0.005 & 3.97 & $2.09-7.52$ & $<0.001$ \\
\hline \multicolumn{7}{|l|}{ Tumor diameter } \\
\hline$\leq 3 \mathrm{~cm}$ & 1.00 (ref.) & & & 1.00 (ref.) & & \\
\hline$>3 \mathrm{~cm}$ & 1.78 & $1.09-2.91$ & 0.021 & 1.99 & $1.16-3.43$ & 0.013 \\
\hline \multicolumn{7}{|l|}{ Histological type } \\
\hline Differentiated & 1.00 (ref.) & & & 1.00 (ref.) & & \\
\hline Undifferentiated & 1.22 & $0.75-1.98$ & 0.417 & 1.31 & $0.77-2.24$ & 0.324 \\
\hline \multicolumn{7}{|l|}{ LBVI } \\
\hline Negative & 1.00 (ref.) & & & 1.00 (ref.) & & \\
\hline Positive & 2.75 & $1.46-5.17$ & 0.002 & 3.85 & $1.75-8.48$ & 0.001 \\
\hline \multicolumn{7}{|l|}{ PNI } \\
\hline Negative & 1.00 (ref.) & & & 1.00 (ref.) & & \\
\hline Positive & 5.12 & $2.76-9.50$ & $<0.001$ & 4.03 & $1.84-8.83$ & $<0.001$ \\
\hline \multicolumn{7}{|l|}{ Resection type } \\
\hline Total & 1.00 (ref.) & & & 1.00 (ref.) & & \\
\hline Subtotal & 0.61 & $0.29-1.28$ & 0.194 & 0.51 & $0.20-1.29$ & 0.155 \\
\hline \multicolumn{7}{|c|}{ Lymph node dissection } \\
\hline D2 & 1.00 (ref.) & & & 1.00 (ref.) & & \\
\hline D1 plus & 1.26 & $0.78-2.05$ & 0.343 & 0.87 & $0.49-1.54$ & 0.625 \\
\hline \multicolumn{7}{|c|}{ Total number of lymph nodes examined } \\
\hline$\geq 15$ & 1.00 (ref.) & & & 1.00 (ref.) & & \\
\hline$<15$ & 1.28 & $0.79-2.08$ & 0.318 & 1.54 & $0.88-2.70$ & 0.129 \\
\hline \multicolumn{7}{|l|}{$\mathrm{aCHT}$} \\
\hline No & 1.00 (ref.) & & & 1.00 (ref.) & & \\
\hline Yes & 0.88 & $0.49-1.59$ & 0.672 & 0.53 & $0.27-1.03$ & 0.061 \\
\hline
\end{tabular}

Abbreviations: OS, overall survival; LBVI, lymphatic and/or blood vessel invasion; PNI, perineural invasion; aCHT, adjuvant chemotherapy 
matching controlled bias. Table 4 shows that patient characteristics were well balanced after matching; and further analysis highlighted that SC group patients received greater survival benefit (HR 0.447, 95\% CI 0.447-0.996, $\mathrm{p}=0.042$; Figure 2).

Toxicity. Toxicity data related to postoperative chemotherapy was collected and here toxicity was defined precisely as in the Common Toxicity Criteria of the National Cancer Institute, version 4.0. There were 19.6\% (10 of 51) patients who suffered from grade 1-2 hematologic toxicity and $7.8 \%$ (4 of 51) had grades 3 and 4. Approximately 21.6\% (11 of 51) of the patients experienced grade 1-2 gastrointestinal reactions, including nausea/vomiting and diarrhea but none developed grade 3 or worse gastrointestinal toxicity. The incidence of grade 1-2 hepatic toxicity, such as elevated AST, ALT and total serum bilirubin level, was $11.7 \%$. Finally, of the 51 patients who received postoperative chemotherapy, 47 continued treatment for at least 3 months (91.2\%).

\section{Discussion}

This retrospective study established the high-risk subgroup of pathological T2N0 gastric cancer patients who could benefit from adjuvant chemotherapy. After careful search, 225 patients with T2N0M0 gastric cancer were included in the final analysis. We identified the following four independent risk factors for OS through the multivariate Cox regression model; tumor location and diameter, LBVI and PNI The patient group with at least one risk factor was defined as the high-risk group and these had significantly lower overall survival than those without risk factors., Further, high-risk patient characteristics were well balanced through propensity score-matched analyses, and significant survival benefit was noted in SC group patients.

Although there is a lack of prospective studies exploring the role of adjuvant therapy in early gastric cancer, there were still some retrospective studies identifying prognostic factors in patients with early gastric cancer. The authors of a Korean study found that the six factors; age, sex, IB stage, lymphatic vessel invasion, nerve invasion, and high serum carcino-embryonic antigen level indices were independent prognostic factors [14]. A further Chinese study reported that lymphatic vessel and nerve invasion and tumor size are independent risk factors [9].

Herein, we identified four independent risk factors (Tumor location, Tumor diameter, LBVI and PNI), and although each of these had previously been individually confirmed, no other study supported our precise risk factor combination. The main reason for this inconsistency was study heterogeneity; with differences in race, surgical practice and initial prognosis.

Do high-risk subgroups benefit from adjuvant therapy? We further explored the effects of adjuvant chemotherapy on survival in our study cohort and observed significant
SC group survival benefit after patient characteristics were well-balanced through propensity score-matched analyses. Four SEER (Surveillance, Epidemiology, and End Results) Program population-based studies have investigated the

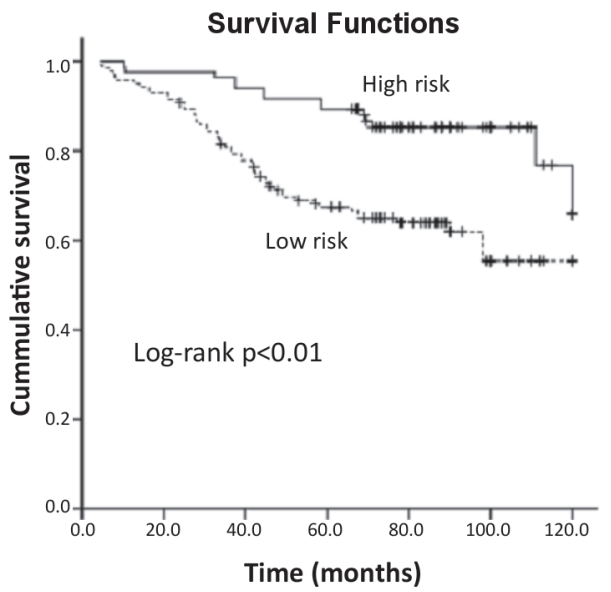

Figure 1. Overall survival in the high risk and low risk subgroups.

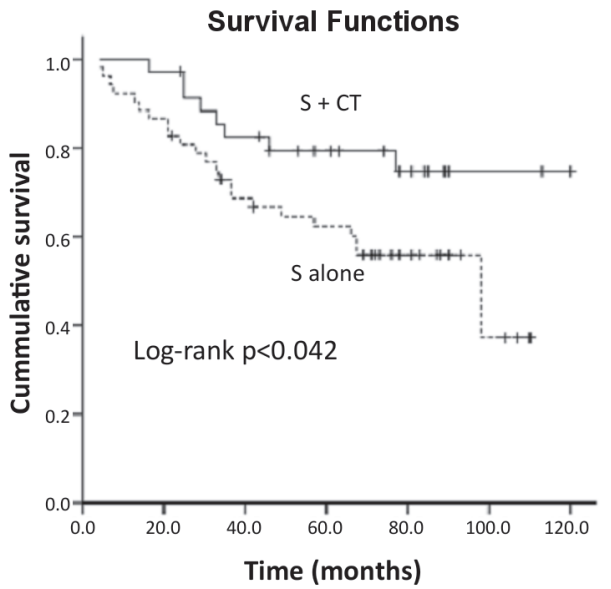

Figure 2. Overall survival in high risk subgroup. S: surgery; S+C: surgery and adjuvant chemotherapy.

Table 3. Univariate analysis for OS in patients undergoing different CHT types.

\begin{tabular}{lcccc}
\hline Characteristics of CHT & $\mathbf{n}$ & HR & 95\% CI & p-value \\
\hline Types of CHT & & & & 0.012 \\
$\quad$ Monotherapy & 6 & 1 & & \\
$\quad$ Combined CHT & 45 & 0.211 & $0.063-0.710$ & \\
CHT regimens & & & & 0.864 \\
$\quad$ Taxane based CHT & 7 & 1 & & \\
$\quad$ Fluorouracil based CHT & 44 & 1.142 & $0.250-5.213$ & \\
CHT cycles & & & & 0.048 \\
$\quad<3$ cycles & 5 & 1 & & \\
$\geq 3$ cycles & 46 & 0.265 & $0.071-0.985$ & \\
\hline
\end{tabular}

Abbreviations: OS, overall survival; CHT, chemotherapy 
Table 4. clinical characteristics of patients in the high risk group before and after propensity score matching.

\begin{tabular}{|c|c|c|c|c|c|c|c|c|}
\hline \multirow{2}{*}{ Variable } & \multicolumn{4}{|c|}{ Before matching } & \multicolumn{4}{|c|}{ After matching } \\
\hline & All Cohort & No aCHT & aCHT & p-value & All Cohort & No aCHT & aCHT & p-value \\
\hline No. of patients & $141(100.0)$ & $99(70.2)$ & $42(29.8)$ & & $87(100.0)$ & $52(50.0)$ & $35(50.0)$ & \\
\hline Age (years) & & & & 0.360 & & & & 0.984 \\
\hline$<50$ & $27(19.1)$ & $17(17.2)$ & $10(23.8)$ & & $15(17.2)$ & $9(17.3)$ & $6(17.1)$ & \\
\hline$\geq 50$ & $114(80.9)$ & $82(82.8)$ & $32(76.2)$ & & $59(82.8)$ & $43(82.7)$ & $29(82.9)$ & \\
\hline Gender & & & & 0.584 & & & & 0.900 \\
\hline Male & $103(73.0)$ & $71(71.7)$ & $32(76.2)$ & & $64(73.6)$ & $38(73.1)$ & $26(74.3)$ & \\
\hline Female & $38(27.0)$ & $28(28.3)$ & $10(23.8)$ & & $23(26.4)$ & $14(26.9)$ & $9(25.7)$ & \\
\hline Tumor location & & & & 0.098 & & & & 0.858 \\
\hline Upper $1 / 3$ & $42(29.8)$ & $34(34.3)$ & $8(19.0)$ & & $18(20.7)$ & $10(19.2)$ & $8(22.9)$ & \\
\hline Middle1/3 & $28(19.9)$ & $16(19.2)$ & $12(28.6)$ & & $17(19.5)$ & $11(21.2)$ & $6(17.1)$ & \\
\hline Lower1/3 & $71(50.4)$ & $49(49.5)$ & $22(50.4)$ & & $52(59.8)$ & $31(59.6)$ & $21(60.0)$ & \\
\hline Tumor diameter & & & & 0.250 & & & & 0.751 \\
\hline$\leq 3 \mathrm{~cm}$ & $36(25.5)$ & $28(28.3)$ & $8(19.0)$ & & $16(18.4)$ & $9(16.3)$ & $7(17.5)$ & \\
\hline$>3 \mathrm{~cm}$ & $105(74.5)$ & $71(71.7)$ & $34(81.0)$ & & $71(81.6)$ & $43(83.7)$ & $28(82.5)$ & \\
\hline Histological type & & & & 0.019 & & & & 0.695 \\
\hline Differentiated & $75(53.2)$ & $59(59.6)$ & $16(38.1)$ & & $37(42.5)$ & $23(44.2)$ & $14(40.0)$ & \\
\hline Undifferentiated & $66(46.8)$ & $40(40.4)$ & $26(61.9)$ & & $50(57.5)$ & $29(55.8)$ & $21(60.0)$ & \\
\hline LBVI & & & & 0.017 & & & & 0.233 \\
\hline Negative & $117(89.3)$ & $87(87.9)$ & $30(71.4)$ & & $70(80.5)$ & $45(84.6)$ & $29(74.3)$ & \\
\hline Positive & $24(710.7)$ & $12(12.1)$ & $12(28.6)$ & & $17(19.5)$ & $7(15.4)$ & $6(25.7)$ & \\
\hline PNI & & & & 0.366 & & & & 0.637 \\
\hline Negative & $123(87.2)$ & $88(88.79)$ & $35(83.3)$ & & $74(85.1)$ & $34(86.5)$ & $35(82.9)$ & \\
\hline Positive & $18(12.8)$ & $11(11.1)$ & $7(16.7)$ & & $13(14.9)$ & $6(13.5)$ & $5(17.1)$ & \\
\hline Resection type & & & & 0.760 & & & & $1.000^{*}$ \\
\hline Subtotal & $133(94.3)$ & $93(93.9)$ & $40(95.2)$ & & $81(93.1)$ & $48(92.3)$ & $33(94.3)$ & \\
\hline Total & $8(5.7)$ & $6(6.1)$ & $2(4.98)$ & & $6(6.9)$ & $4(7.7)$ & $2(5.7)$ & \\
\hline Lymph node dissection & & & & 0.862 & & & & 0.510 \\
\hline D2 & $79(56.0)$ & $55(55.6)$ & $24(57.1)$ & & $46(52.9)$ & $29(55.8)$ & $17(48.6)$ & \\
\hline D1 plus & $62(44.0)$ & $44(44.4)$ & $18(42.9)$ & & $41(47.1)$ & $23(44.2)$ & $18(51.4)$ & \\
\hline $\begin{array}{l}\text { Total number of lymph } \\
\text { nodes examined }\end{array}$ & & & & 0.367 & & & & 0.753 \\
\hline$\geq 15$ & $72(51.1)$ & $53(53.5)$ & $19(45.2)$ & & $38(43.7)$ & $22(42.3)$ & $16(45.7)$ & \\
\hline$<15$ & $69(48.9)$ & $46(46.5)$ & $23(54.8)$ & & $49(56.3)$ & $30(57.7)$ & $19(54.3)$ & \\
\hline
\end{tabular}

* Fisher's Exact Test; Abbreviations: LBVI, lymphatic and/or blood vessel invasion; PNI, perineural invasion; aCHT, adjuvant chemotherapy

role of adjuvant therapy in early gastric cancer. One study reported that adjuvant radiotherapy provided survival benefits to T2N0 GC patients with $<15$ lymph nodes, but no survival benefit for adjuvant chemotherapy was identified [15]. Two other studies reported survival advantage in chemo-radiotherapy for locally advanced GC (stage II and III), but no stage I survival benefit was found $[7,8]$.

These results are inconsistent with our findings for adjuvant chemotherapy in early high-risk patients. We noted that important risk factors in our study such as lymphatic and/or blood vessel invasion (LBVI negative and positive) and perineural invasion (PNI negative and positive) were not included in their analysis; LBVI has always been considered the commencement of lymphogenous or hematogenous metastasis and PNI is a further route of tumor spread. LBVI and PNI were not only demonstrated to be poor independent prognostic markers for survival, but also markers for adjuvant chemotherapy in various kinds of solid tumor. Therefore, research inconsistency resulted from the lack of these important risk factors in other studies. Although the fourth SEER study also showed survival benefit of adjuvant chemo-radiotherapy following gastrectomy, unfortunately it did not perform a stratified analysis by tumor stage [16].

Since 1997, retrieval of at least 15 lymph nodes has been recommended for adequate gastric cancer staging and several studies have found that lymphadenectomy with $<15$ lymph nodes removed was an adverse independent prognostic factor for overall survival. For example, Haejin (2016) found that their subgroup of patients with suboptimal lymphadenectomy benefitted from chemo-radiotherapy rather than 
chemotherapy [15]. Although differences in radiotherapy and chemo-radiotherapy roles could not be established in our study because of the lack of patients receiving postoperative adjuvant radiotherapy, we did not find that the number of dissected lymph node affected survival. This is supported by the 2001 Chinese study [9] which also failed to show that the number of removed lymph nodes was an independent prognostic factor, and similar results were also observed in a further SEER based study [7].

We speculate the following reasons for this disparity; (1) early gastric cancer has less metastatic lymph nodes and lower requirement for the number of removed lymph nodes than locally advanced gastric cancer and (2) LBVI and PNI were not analyzed in these studies; and LBVI and PNI could easily offset the reported prognostic effects of the number of lymph nodes removed.

However, there are several potential limitations in our study. As previously noted, radiotherapy and chemo-radiotherapy roles were not analyzed due to lack of patients receiving postoperative adjuvant radiotherapy. Therefore, adjuvant radiotherapy benefit for T2N0 GC who underwent surgery needs further investigation. In addition, this is a retrospective study, and although there are likely patient and tumor baseline characteristic imbalances between $t$ treatment groups, we performed propensity score-matched analyses to control for baseline confounders and reduce bias. Finally, the number of patients with T2N0 gastric cancer was relatively small and resultant effects may be underestimated and results should be interpreted with caution. We note here that a prospective randomized trial comparing sole surgery in stage I GC patients at high risk of recurrence or death with their adjuvant chemotherapy is now ongoing [17] and this prospective trial with large sample size is expected to compensate for previous research shortcomings and yield satisfactory results.

In conclusion, we identified a high-risk subgroup of T2N0 gastric cancer patients with at least one of the following independent risk factors; tumor location in the upper $1 / 3$ of the stomach, larger tumor diameter, lymphatic and/or blood vessel invasion and perineural invasion. The adjuvant chemotherapy provided to this subgroup significantly improved their overall survival and our findings are expected to be supported by future prospective trials.

\section{References}

[1] FERLAY J, SOERJOMATARAM I, ERVIK M, FORMAN D, BRAY F et al. Globocan 2012: Estimated cancer incidence, mortality and prevalence in 2012. IARC 2018. http://globocan.iarc.fr

[2] SAKURAMOTO S, SASAKO M, YAMAGUCHI T, KINOSHITA T, FUJII $M$ et al. Adjuvant chemotherapy for gastric cancer with s-1, an oral fluoropyrimidine. N Eng J Med 2007; 357: 1810-1820. https://doi.org/10.1056/NEJMoa072252
[3] GROUP G, PAOLETTI X, OBA K, BURZYKOWSKI T, MICHIELS $S$ et al. Benefit of adjuvant chemotherapy for resectable gastric cancer: a meta-analysis. JAMA 2010; 303: 1729-1737. https://doi.org/10.1001/jama.2010.534

[4] NOH SH, PARK SR, YANG HK, CHUNG HC, CHUNG IJ et al. Adjuvant capecitabine plus oxaliplatin for gastric cancer after d2 gastrectomy (classic): 5-year follow-up of an open-label, randomised phase 3 trial. Lancet Oncol 2014; 15: 1389-1396. https://doi.org/10.1016/S1470-2045(14)70473-5

[5] NAKAJIMA T, NASHIMOTO A, KITAMURA M, KITO T, IWANAGA $\mathrm{T}$ et al. Adjuvant mitomycin and fluorouracil followed by oral uracil plus tegafur in serosa-negative gastric cancer: A randomised trial. Gastric cancer surgical study group. Lancet 1999; 354: 273-277.

[6] JIMENO-ARANDA A, SAINZ SAMITIER R ARAGUES GM. Gastric cancer in the province of Zaragoza (Spain): a survival study. Neoplasma 1996; 43: 199-203.

[7] COBURN NG, GOVINDARAJAN A, LAW CH, GULLER U, KISS A et al. Stage-specific effect of adjuvant therapy following gastric cancer resection: A population-based analysis of 4,041 patients. Ann Surg Oncol 2008; 15: 500-507. https:// doi.org/10.1245/s10434-007-9640-0

[8] SEYEDIN S, WANG PC, ZHANG Q LEE P. Benefit of adjuvant chemoradiotherapy for gastric adenocarcinoma: A seer population analysis. Gastrointest Cancer Res 2014; 7: 82-90.

[9] DU C, ZHOU Y, HUANG K, ZHAO G, FU H et al. Defining a high-risk subgroup of pathological $\mathrm{t} 2 \mathrm{n} 0$ gastric cancer by prognostic risk stratification for adjuvant therapy. J Gastrointest Surg 2011; 15: 2153-2158. https://doi.org/10.1007/ s11605-011-1684-6

[10] JIN LX, MOSES LE, SQUIRES III MH, POULTSIDES GA, VOTANOPOULOS $\mathrm{K}$ et al. Factors associated with recurrence and survival in lymph node-negative gastric adenocarcinoma: A 7-institution study of the us gastric cancer collaborative. Ann Surg 2015; 262: 999-1005. https://doi. org/10.1097/SLA.0000000000001084

[11] PARK SY, LEE JG, KIM J, BYUN GE, BAE MK et al. Efficacy of platinum-based adjuvant chemotherapy in t2an0 stage $\mathrm{ib}$ non-small cell lung cancer. J Cardiothorac Surg 2013; 8: 151. https://doi.org/10.1186/1749-8090-8-151

[12] NIKBERG M, CHABOK A, LETOCHA H, KINDLER C, GLIMELIUS B et al. Lymphovascular and perineural invasion in stage ii rectal cancer: A report from the swedish colorectal cancer registry. Acta Oncol 2016; 55: 1418-1424. https://doi.org/10.1080/0284186X.2016.1230274

[13] MAVROUDIS D, SALOUSTROS E, BOUKOVINAS I, PAPAKOTOULAS P, KAKOLYRIS $S$ et al. Sequential vs concurrent epirubicin and docetaxel as adjuvant chemotherapy for high-risk, node-negative, early breast cancer: an interim analysis of a randomised phase iii study from the hellenic oncology research group. Br J Cancer 2017; 117: 164-170. https://doi.org/10.1038/bjc.2017.158

[14] PARK JH, RYU MH, KIM HJ, RYOO BY, YOO C et al. Risk factors for selection of patients at high risk of recurrence or death after complete surgical resection in stage i gastric cancer. Gastric Cancer 2016; 19: 226-233. https://doi. org/10.1007/s10120-015-0464-5 
[15] IN H, KANTOR O, SHARPE SM, BAKER MS, TALAMONTI $\mathrm{MS}$ et al. Adjuvant therapy improves survival for $\mathrm{t} 2 \mathrm{n} 0$ gastric cancer patients with sub-optimal lymphadenectomy. Ann Surg Oncol 2016; 23: 1956-1962. https://doi.org/10.1245/ s10434-015-5075-1

[16] SNYDER RA, CASTALDO ET, BAILEY CE, PHILLIPS SE, CHAKRAVARTHY AB et al. Survival benefit of adjuvant radiation therapy for gastric cancer following gastrectomy and extended lymphadenectomy. Int J Surg Oncol 2012; 2012: 307670. https://doi.org/10.1155/2012/307670
[17] RYU MH, CHO JY, ZANG DY, LEE WK, LEE KW et al. A randomized phase iii study of adjuvant capecitabine vs observation in curatively resected stage IB (by AJCC 6th edition) gastric cancer (CATALYSIS; KCSG ST14-05). J Clin Oncol, 2015; 33 https://doi.org/10.1200/jco.2015.33.15_suppl.tps4137 